\title{
A Novel 3D Imaging Method for Airborne Downward-Looking Sparse Array SAR Based on Special Squint Model
}

\author{
Xiaozhen Ren, Yao Qin, and Lihong Qiao \\ College of Information Science and Engineering, Henan University of Technology, Zhengzhou 450001, China \\ Correspondence should be addressed to Xiaozhen Ren; rxz235@163.com
}

Received 30 June 2014; Revised 4 December 2014; Accepted 18 December 2014; Published 31 December 2014

Academic Editor: Francisco Falcone

Copyright (C) 2014 Xiaozhen Ren et al. This is an open access article distributed under the Creative Commons Attribution License, which permits unrestricted use, distribution, and reproduction in any medium, provided the original work is properly cited.

\begin{abstract}
Three-dimensional (3D) imaging technology based on antenna array is one of the most important 3D synthetic aperture radar (SAR) high resolution imaging modes. In this paper, a novel 3D imaging method is proposed for airborne down-looking sparse array SAR based on the imaging geometry and the characteristic of echo signal. The key point of the proposed algorithm is the introduction of a special squint model in cross track processing to obtain accurate focusing. In this special squint model, point targets with different cross track positions have different squint angles at the same range resolution cell, which is different from the conventional squint SAR. However, after theory analysis and formulation deduction, the imaging procedure can be processed with the uniform reference function, and the phase compensation factors and algorithm realization procedure are demonstrated in detail. As the method requires only Fourier transform and multiplications and thus avoids interpolations, it is computationally efficient. Simulations with point scatterers are used to validate the method.
\end{abstract}

\section{Introduction}

Traditional synthetic aperture radar (SAR) utilizes pulse compression and synthetic aperture technique to form high resolution two-dimensional (2D) images of the observed area with weather independence and all-day operation capabilities $[1,2]$. However, traditional 2D SAR works in side-looking or squint mode and cannot obtain the $3 \mathrm{D}$ information of the scene. Compared with 2D SAR, 3D SAR has distinct advantages in ground topography and forest height estimation and for solving layover effect in natural or urban areas. Multibaseline SAR tomography is an advanced 3D SAR imaging mode, which exploits the multibaseline nature to allow distinguishing multiple scatterers at different heights within the same azimuth-range resolution cell. Therefore, it allows measuring the scattering distribution in the 3D space. Unfortunately, for the current SAR tomography, it is almost impossible to avoid an uneven track distribution in repeatpass data acquisition, which is just the main reason for the strong ambiguity in height $[3,4]$.

Down-looking array SAR is an innovative imaging mode, which was first introduced by Gierull in 1999 [5]. In down-looking array SAR, 3D resolutions are obtained by applying pulse compression technique in range direction, virtual aperture synthesis principle in azimuth direction, and linear array aperture synthesis in cross track direction [6-8]. Down-looking array SAR can overcome restrictions of shading and lay over effects in side-looking SAR and also avoid the height ambiguity problem in SAR tomography caused by the uneven track distribution. However, down-looking array SAR requires more $\mathrm{A} / \mathrm{D}$ sampling devices and data collection devices for echo recording than in traditional SAR. Therefore, sparse linear array with multiple-input-multipleoutput (MIMO) mode is often used in down-looking array SAR, which helps to reduce the complexity of the system $[9,10]$.

Due to its advanced performance, down-looking array SAR has attracted wide attention. Two airborne downlooking array SAR systems, DRIVE [11] and ARTINO [6], are being developed at ONERA and FGANFHR, respectively. Klare [6] transformed the bistatic configuration to monostatic one based on the equivalent phase center principle and proposed a 3D imaging algorithm for cross track imaging using beamforming operation. However, range cell migration 
is not considered. Du et al. [12] made an improvement of the method proposed in [6]. They added the range migration correction in the imaging processing to expand the imaging scene width in the cross track direction. However, the digital beamforming technology used in the paper requires the point by point calculations and inserting the point spread function for focusing the elevation angle. Therefore, this method suffers from severe computational complexity. Giret et al. [13] made some simplifications to the dual square roots with Fresnel approximation and proposed an algorithm derived from that of monostatic configuration. A shortcoming of the algorithm is the range error caused by Fresnel approximation. Subsequently, an accurate 3D focused algorithm based on range migration algorithm (RMA) was presented in [7] but needs $3 \mathrm{D}$ stolt interpolation. In a recent work, some methods based on compressive sensing (CS) were applied to array SAR imaging $[14,15]$, providing an improved resolution in cross track direction. However, these methods suffered from severe computation loads and were hard to realize real-time imaging.

The main topic of this paper is to introduce an accurate and efficient 3D imaging algorithm for down-looking sparse array SAR. According to the analysis of the spatial geometry and the echo signal model, a special squint model is introduced for cross track focusing. The phase compensation factors and algorithm realization procedure are demonstrated in detail.

The rest of the paper is organized as follows. Section 2 presents the geometry and principle of airborne downlooking sparse array SAR system. In Section 3, a novel 3D imaging algorithm for down-looking sparse array SAR is described in detail. The performance of the method is investigated in Section 4. Finally, Section 5 gives a brief conclusion.

\section{Geometrical Model of Airborne Down-Looking Sparse Array SAR}

Figure 1 shows the geometry of down-looking sparse array SAR [15]. $x$ denotes the azimuth direction, $y$ denotes the cross track direction, and $z$ denotes the height direction. The radar platform flies at height $H$ along the $x$-axis with velocity $v$. The sparse linear array is mounted in the cross track direction along the wings and is designed based on ARTINO array distribution. That is because that ARTINO array distribution is quite easy for hardware implementation [16]. The sparse linear array composes $M$ transmitting antenna elements and $N$ receiving antenna elements and works in the time division mode. Each time, only one transmitting antenna element transmits signal and all the receiving antenna elements receive echo simultaneously [6]. The transmitting antenna elements work sequentially and an aperture synthesis period is acquired until all the transmitting antenna elements have worked for once.

According to the principle of equivalent phase center, the sparse linear array formed by the ARTINO array distribution can be equivalent to a virtual uniform linear array $[6,10]$.

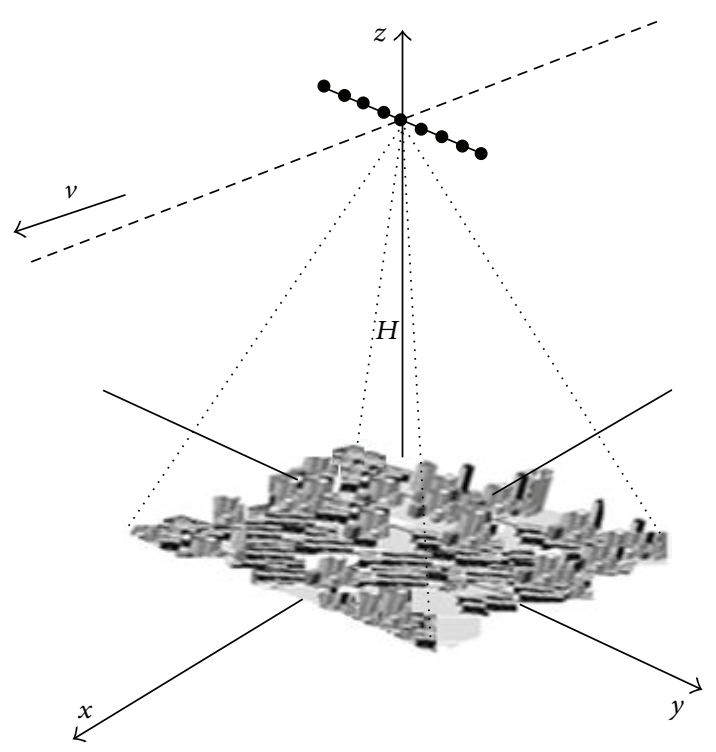

FIGURE 1: Geometry of down-looking sparse array SAR.

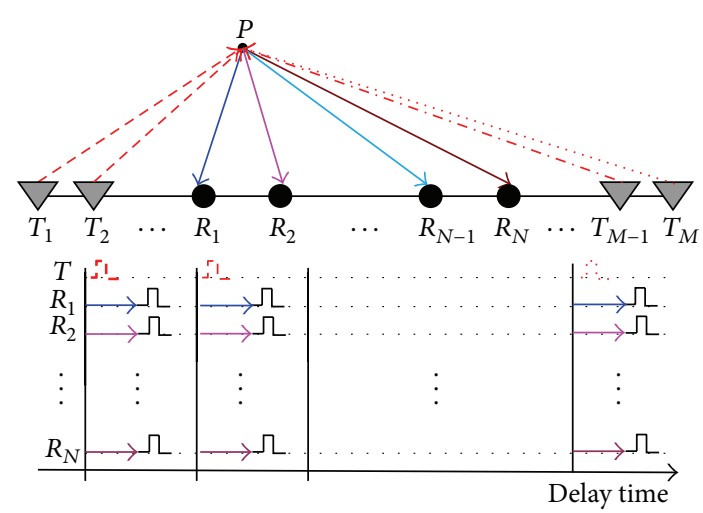

FIGURE 2: Transmitting and receiving order of down-looking sparse array SAR.

The virtual antenna array is composed of $M N$ virtual elements and each virtual element transmits and receives by itself. These virtual antenna elements are uniformly distributed along the wings and centered at the $y$-axis. Each virtual antenna element is located at the mean position of a real single transmitting element and a real single receiving element. Figure 2 shows the transmitting and the receiving order of each antenna element for a down-looking sparse array SAR. $P$ is the target in the imaging scene, $T_{i}$ with $i \in[1, M]$ denotes the transmitting antenna element, and $R_{j}$ with $j \in[1, N]$ denotes the receiving antenna element. As shown in Figure 2, the transmitting antenna elements work sequentially. The antenna element $T_{1}$ transmits signal first and all the receiving antenna elements receive echo simultaneously. Then the antenna element $T_{2}$ transmits signal, and the procedure loops until the entire transmitting antenna elements have worked for once, and then an aperture synthesis period is acquired. 


\section{Three-Dimensional Imaging Algorithm for Airborne Down-Looking Sparse Array SAR}

Based on the principle of equivalent phase center, the sparse linear array formed by the time division mode is equal to a virtual linear array, and each virtual antenna element transmits and receives signal by itself.

3.1. Equivalent Phase Error Compensation. Consider the data acquisition shown in Figure 1 . At the slow time $\tau_{m}$, the positions of the transmitting and receiving antenna elements are given by $\left(x, y_{T i}, H\right)$ and $\left(x, y_{R j}, H\right)$, respectively. Then the transmitting and receiving paths $R_{T i}$ and $R_{R j}$ of the point scatterer $P$ positioned at $\left(x_{p}, y_{p}, z_{p}\right)$ are given by

$$
\begin{aligned}
& R_{T i}=\sqrt{\left(x-x_{p}\right)^{2}+\left(y_{T i}-y_{p}\right)^{2}+\left(H-z_{p}\right)^{2}}, \\
& R_{R j}=\sqrt{\left(x-x_{p}\right)^{2}+\left(y_{R j}-y_{p}\right)^{2}+\left(H-z_{p}\right)^{2}},
\end{aligned}
$$

where $x=v \tau_{m}$ denotes the azimuth position and $y_{T i}$ and $y_{R j}$ denote the cross track positions of the $i$ th transmitting antenna element and the $j$ th receiving antenna element, respectively.

Then the sum of the transmitting and receiving paths of the point scatterer $P$ contains two square roots, which will lead to complex computation for the following imaging process. When the distance between the transmitting and receiving antenna elements is far less than the observation distance of the radar, the above complete travelling path can be equal to the dual echo paths from the virtual antenna element located at $y=\left(y_{T i}+y_{R j}\right) / 2$ to point scatterer $P$ based on the principle of equivalent phase center [6]. And the equivalent echo path can be written as

$$
R=\sqrt{\left(x-x_{p}\right)^{2}+\left(y-y_{p}\right)^{2}+\left(H-z_{p}\right)^{2}} .
$$

Then, the phase difference between the virtual antenna element and the reality antenna element should be compensated by

$$
\Delta \varphi=\frac{2 \pi}{\lambda}\left(R_{i, j}-2 R\right) \approx \frac{\pi\left(y_{T i}-y_{R j}\right)^{2}}{2 \lambda R_{P}},
$$

where $\lambda$ is the wavelength and $R_{P}=\sqrt{\left(x-x_{p}\right)^{2}+\left(H-z_{p}\right)^{2}}$.

3.2. Motion Compensation. Furthermore, the airborne downlooking sparse array SAR works in the time division mode; the virtual antenna elements obtained from different pulse repetition period are not in a straight line with the movement of the platform. Therefore, in order to obtain a fully distributed virtual uniform linear array, the motion compensation should be implemented. The compensated phase caused by the overtake or lag phases owing to the position difference of the antenna elements is given by

$$
\begin{aligned}
& \Delta \varphi_{l, k} \\
& =\frac{4 \pi}{\lambda}\left(\sqrt{\left(\frac{M v}{\mathrm{PRF}}\left(l-\frac{L}{2}\right)+\Delta x_{k}-x_{p}\right)^{2}+\left(y-y_{p}\right)^{2}+\left(H-z_{p}\right)^{2}}\right. \\
& \left.-\sqrt{\left(\frac{M v}{\mathrm{PRF}}\left(l-\frac{L}{2}\right)-x_{p}\right)^{2}+\left(y-y_{p}\right)^{2}+\left(H-z_{p}\right)^{2}}\right) \\
& l=1,2, \ldots, L ; \quad k=1,2, \ldots, M,
\end{aligned}
$$

where $L$ is the azimuth sample number and $\Delta x_{k}$ is the move spacing between the $k$ th and the first transmitting antenna element in the azimuth direction. $v$ is the velocity of the platform and PRF is the pulse repetition frequency.

3.3. Echo Signal. After the process above, the collected data of airborne down-looking sparse array SAR can be regarded as received by the fully distributed virtual linear array. For an arbitrary point scatterer positioned at $P\left(x_{p}, y_{p}, z_{p}\right)$, the distance between the $m$ th virtual antenna element and the point scatterer is given by

$$
\begin{aligned}
R & =\sqrt{\left(v \tau-x_{p}\right)^{2}+\left(y_{m}-y_{p}\right)^{2}+\left(H-z_{p}\right)^{2}} \\
& =\sqrt{\left(v \tau-x_{p}\right)^{2}+R_{B}^{2}},
\end{aligned}
$$

where $\tau$ is the azimuth time and $R_{B}=$ $\sqrt{\left(H-z_{p}\right)^{2}+\left(y_{m}-y_{p}\right)^{2}}$. The echo signal received by the $m$ th virtual antenna element can be written as

$$
\begin{aligned}
s_{1}\left(\hat{t}, \tau, y_{m}\right)= & a_{r}\left(\hat{t}-\frac{2 R}{c}\right) a_{a}\left(\tau-\tau_{p}\right) \\
& \times \exp \left[j \pi \gamma\left(\hat{t}-\frac{2 R}{c}\right)^{2}-j \frac{4 \pi}{\lambda} R\right],
\end{aligned}
$$

where $\hat{t}$ denotes the fast time, $y_{m}$ denotes the cross track position of the $m$ th virtual antenna element, $\tau_{p}=x_{p} / v$ is the azimuth time of the point scatterer $P, c$ is the light velocity, $\gamma$ is the range frequency rate, $a_{r}(\cdot)$ is the rectangular window function in range direction, and $a_{a}(\cdot)$ is the rectangular window function in azimuth direction.

3.4. Azimuth and Range Compression. As the signal received by each virtual antenna element in the airborne downlooking sparse array SAR can be treated as the echo data received in side-looking mode, the classical $2 \mathrm{D}$ imaging methods can be used to process the data received by each virtual antenna element, which can be found in $[17,18]$. 


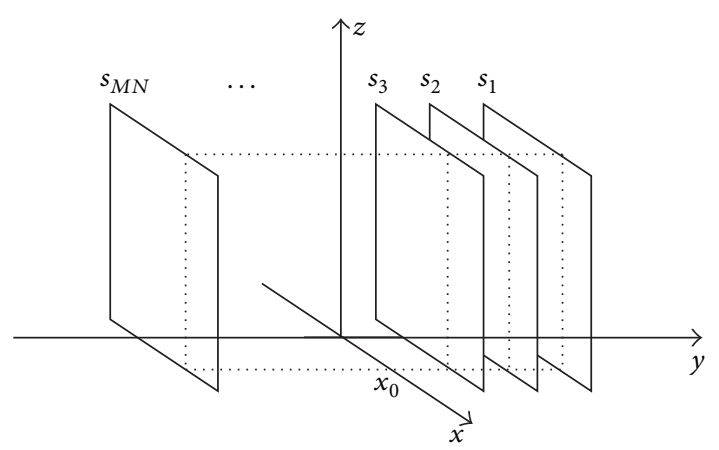

FIgURE 3: 2D focused images in cross track direction of downlooking sparse array SAR.

Consequently, a SAR image corresponding to the $m$ th virtual antenna element, which is obtained after the azimuth and range direction focusing, can be expressed as $[17,18]$

$$
\begin{aligned}
s\left(\hat{t}, \tau, y_{m}\right)= & A \operatorname{sinc}\left[B_{r}\left(\hat{t}-\frac{2 R_{B}}{c}\right)\right] \operatorname{sinc}\left[B_{a}\left(\tau-\tau_{p}\right)\right] \\
& \times \exp \left(-j \frac{4 \pi R_{B}}{\lambda}\right)
\end{aligned}
$$

where $A$ is the amplitude of the focused point target, $B_{r}$ is the range bandwidth, and $B_{a}$ is the azimuth bandwidth.

3.5. Cross Track Compression. Suppose that the signal received by each virtual antenna element have been focused by range-Doppler algorithm, and then $M N$ 2D SAR images can be obtained in cross track direction, as shown in Figure 3. Here $M N$ is the number of the virtual antenna elements. If all the SAR images have been coregistered first, then, in the imaging area, the azimuth positions of each point target in all SAR images are invariable, while only the distances between the virtual antenna elements and the point target vary with the positions of the virtual antenna elements. Therefore, a range-cross track section corresponding to one azimuth position can be focused at a time. Then the 3D image of airborne down-looking array SAR can be obtained by processing all the sections with the same procedure.

Then the range-cross track section corresponding to azimuth time $\tau=\tau_{0}$ can be written as

$$
\begin{aligned}
s\left(\hat{t}, y_{m} ; \tau=\tau_{0}\right)= & A \operatorname{sinc}\left[B_{r}\left(\hat{t}-\frac{2 R_{B}}{c}\right)\right] \\
& \times \operatorname{sinc}\left[B_{a}\left(\tau-\tau_{p}\right)\right] \exp \left(-j \frac{4 \pi R_{B}}{\lambda}\right) \\
= & A_{1} \operatorname{sinc}\left[B_{r}\left(\hat{t}-\frac{2 R_{B}}{c}\right)\right] \exp \left(-j \frac{4 \pi R_{B}}{\lambda}\right),
\end{aligned}
$$

where $A_{1}=A \operatorname{sinc}\left[B_{a}\left(\tau_{0}-\tau_{p}\right)\right]$. As $A_{1}$ has no effect on the imaging process, it will be ignored in the following analysis. The slant range between the $m$ th virtual antenna element and

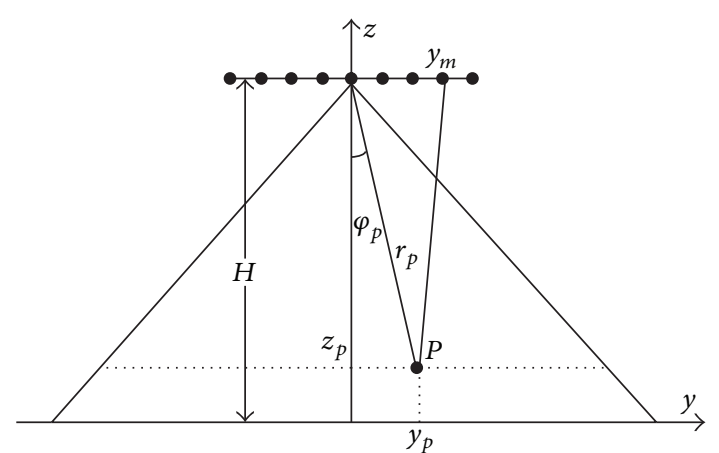

FIGURE 4: Geometry relations in cross track-height section.

the point target positioned at $\left(x_{p}, y_{p}, z_{p}\right)$ in this section is given by

$$
R_{B}=\sqrt{\left(H-z_{p}\right)^{2}+\left(y_{m}-y_{p}\right)^{2}} .
$$

From (8) we can get that target responses take on sinc function, with positions of peak value distributing along the range trajectories in the cross track direction. To simplify further analysis, we redraw the geometry relations of the cross track-height section in Figure 4. It can be seen that as the real antenna array length is far less than the imaging size in cross track direction, for the point targets located in the same height position, the range trajectories and scanning angles are different from each other. That is different from the characteristic of SAR working in squint mode, as the point targets located in the same height position have the same range trajectories and scanning angles for conventional squint SAR. Therefore, we introduce a special squint model for the cross track imaging in airborne down-looking array SAR. In this special squint model, the distance between the point target and the center of the antenna array is defined as the equivalent squint range $r_{p}$, and the corresponding scanning angle is defined as equivalent squint angle $\varphi_{p}$. Then, from Figure 4, we can get that the point targets with different cross track positions have different squint angles and squint ranges at the same height in this special squint model. And the slant range between the $m$ th virtual antenna element and the point target positioned at $\left(y_{p}, z_{p}\right)$ can also be rewritten as

$$
\begin{aligned}
R_{B} & =\sqrt{\left(H-z_{p}\right)^{2}+\left(y_{m}-y_{p}\right)^{2}} \\
& =\sqrt{r_{p}^{2}+y_{m}^{2}-2 r_{p} y_{m} \sin \varphi_{p}},
\end{aligned}
$$

where

$$
r_{p}=\frac{\left(H-z_{p}\right)}{\cos \varphi_{p}}
$$

As the target responses distribute along the range trajectories in the cross track direction. In order to concentrate energy to realize focusing in the cross track direction, work 
only has to be done along this trajectory. However, the range trajectories of point targets at different heights are interlaced, and the cross track focusing is difficult to realize in time domain. Transform the signal expressed in (8) into the rangecross track $2 \mathrm{D}$ frequency domain via the stationary phase method. Then, we have

$$
\begin{aligned}
S\left(f_{r}, f_{y} ; \tau=\tau_{0}\right)= & a_{r}\left(-\frac{f_{r}}{\gamma_{e}\left(f_{y} ; R_{B}\right)}\right) \\
& \times \exp \left(-j 2 \pi f_{y} y_{p}\right) \exp \left[\theta\left(f_{r}, f_{y}\right)\right],
\end{aligned}
$$

where $f_{r}$ is the range frequency, $f_{y}$ is the cross track frequency, and the range frequency rate is changed from $\gamma$ to $\gamma_{e}\left(f_{y} ; R_{B}\right)$ in cross track frequency domain, which is dependent on cross track frequency and on range

$$
\frac{1}{\gamma_{e}\left(f_{y} ; R_{B}\right)}=\frac{1}{\gamma}-\frac{\lambda^{3} R_{B} f_{y}^{2}}{2 c^{2}\left[1-\left(\lambda f_{y} / 2\right)^{2}\right]^{3 / 2}}
$$

And the phase function $\theta\left(f_{r}, f_{y}\right)$ is expressed as

$$
\theta\left(f_{r}, f_{y}\right)=-j \frac{4 \pi r_{p} \cos \varphi_{p}}{\lambda} \sqrt{\left(1+\frac{f_{r}}{f_{c}}\right)^{2}-\left(\frac{\lambda f_{y}}{2}\right)^{2}} .
$$

To aid further analysis, $\theta\left(f_{r}, f_{y}\right)$ is expanded into Taylor's series

$$
\begin{aligned}
\theta\left(f_{r}, f_{y}\right)= & \theta\left(f_{r}=0, f_{y}\right)+\left.f_{r} \frac{d \theta\left(f_{r}, f_{y}\right)}{d f_{r}}\right|_{f_{r}=0} \\
& +\left.\frac{f_{r}^{2}}{2 !} \frac{d^{2} \theta\left(f_{r}, f_{y}\right)}{d f_{r}^{2}}\right|_{f_{r}=0}+\left.\frac{f_{r}^{3}}{3 !} \frac{d^{3} \theta\left(f_{r}, f_{y}\right)}{d f_{r}^{3}}\right|_{f_{r}=0} \\
& +\cdots \\
= & -j \frac{4 \pi r_{p} \cos \varphi_{p}}{\lambda} \alpha\left(f_{y}\right)-j \frac{4 \pi r_{p} \cos \varphi_{p}}{c \alpha\left(f_{y}\right)} f_{r} \\
& +j \frac{\pi r_{p} \cos \varphi_{p} c f_{y}^{2}}{2 f_{c}^{3} \alpha^{3}\left(f_{y}\right)} f_{r}^{2}-j \frac{\pi r_{p} \cos \varphi_{p} c f_{y}^{2}}{2 f_{c}^{4} \alpha^{5}\left(f_{y}\right)} f_{r}^{3} \\
& +\cdots,
\end{aligned}
$$

where

$$
\alpha\left(f_{y}\right)=\sqrt{1-\left(\frac{\lambda f_{y}}{2}\right)^{2}}
$$

In (15), the first term represents the modulation in cross track direction, determining the cross track compression; the second term contains the information of range migration, reflecting the target position in the range direction; the third and fourth terms are the secondary range compression item, showing the coupling of range and cross track in the signal spectrum; the other higher order terms (power is greater than three) are very small and thus ignorable.

From (15) we can get that the range migration and secondary range compression items depend on the equivalent squint range $r_{p}$ and squint angle $\varphi_{p}$ of the point target, and the equivalent squint ranges and squint angles vary with the positions of point targets. However, from Figure 4, we can get that when the targets are positioned at the same height, the term $r_{p} \cos \varphi_{p}$ is a constant, which is irrelevant to the target positions and is equal to the shortest distance from the targets to the antenna array. Therefore, the range migration and secondary range compression items can be compensated uniformly, which can be constructed with target positions independence.

From (15), the reference function for range migration correction can be written as

$$
H_{\mathrm{RCM}}\left(f_{r}, f_{y}\right)=\exp \left[j \frac{4 \pi}{c}\left(\frac{1}{\alpha\left(f_{y}\right)}-1\right)\left(H-z_{p}\right) f_{r}\right]
$$

And the reference function for secondary range compression can be written as

$$
\begin{aligned}
& H_{\mathrm{SRC}}\left(f_{r}, f_{y}\right) \\
& \quad=\exp \left[-j \frac{\pi c\left(H-z_{p}\right) f_{y}^{2}}{2 f_{c}^{3} \alpha^{3}\left(f_{y}\right)} f_{r}^{2}+j \frac{\pi c\left(H-z_{p}\right) f_{y}^{2}}{2 f_{c}^{4} \alpha^{5}\left(f_{y}\right)} f_{r}^{3}\right] .
\end{aligned}
$$

From (17) and (18), we get that the range migration correction term and secondary range compression term are dependent on the height of the target. In the real case, we use the slant range $R_{B}$ to replace the range $\left(H-z_{p}\right)$ in the imaging processing when the scene width is relatively less than the distance between the platform and the target.

After the range migration correction and secondary range compression, the range trajectory of each point target will be corrected to a line approximately. Transform the signal into the range time-cross track frequency domain, and the range compression signal is given by

$$
\begin{aligned}
s\left(\hat{t}, f_{y} ; \tau=\tau_{0}\right)= & \operatorname{sinc}\left[B_{r}\left(\widehat{t}-\frac{2\left(H-z_{p}\right)}{c}\right)\right] \\
& \times \exp \left(-j 2 \pi f_{y} y_{p}\right) \\
& \times \exp \left[-j \frac{4 \pi r_{p} \cos \varphi_{p}}{\lambda} \alpha\left(f_{y}\right)\right] .
\end{aligned}
$$


TABLE 1: Parameters used for simulation.

\begin{tabular}{lc}
\hline Parameter & Value \\
\hline Carrier frequency & $37.5 \mathrm{GHz}$ \\
Pulse bandwidth & $300 \mathrm{MHz}$ \\
Pulse repetition frequency & $1024 \mathrm{~Hz}$ \\
Chirp duration & $1.0 \mu \mathrm{s}$ \\
Radar height & $500 \mathrm{~m}$ \\
Radar velocity & $50 \mathrm{~m} / \mathrm{s}$ \\
Number of transmitting antenna elements & 4 \\
Number of receiving antenna elements & 32 \\
Azimuth beam width & $0.57^{\circ}$ \\
Cross track beam width & $12^{\circ}$ \\
\hline
\end{tabular}

TABLE 2: The position parameters of the point targets.

\begin{tabular}{lccc}
\hline Point targets & Azimuth $(\mathrm{m})$ & Range $(\mathrm{m})$ & Cross track $(\mathrm{m})$ \\
\hline 1 & 0 & 490 & 10 \\
2 & 4 & 495 & 20 \\
3 & 4 & 495 & -20 \\
4 & -4 & 495 & 20 \\
5 & -4 & 495 & -20 \\
6 & 8 & 500 & 40 \\
7 & 8 & 500 & -40 \\
8 & -8 & 500 & 40 \\
9 & -8 & 500 & -40 \\
\hline
\end{tabular}

The next step is to proceed to the cross track focusing. Transform the signal (19) into the range-cross track 2D time domain

$$
\begin{aligned}
s\left(\widehat{t}, y_{m} ; \tau=\tau_{0}\right)= & \operatorname{sinc}\left[B_{r}\left(\widehat{t}-\frac{2\left(H-z_{p}\right)}{c}\right)\right] \\
& \times \exp \left[-j \frac{2 \pi\left(y_{m}-y_{p}\right)^{2}}{\lambda r_{p} \cos \varphi_{p}}\right] \\
& \times \exp \left(-j \frac{4 \pi r_{p} \cos \varphi_{p}}{\lambda}\right) .
\end{aligned}
$$

The first phase term in (20) represents a quadratic distortion, which can be compensated by deramp processing. And the quadratic phase reference function for cross track deramp processing is

$$
H_{\text {Deramp }}\left(y_{m}\right)=\exp \left(j \frac{2 \pi y_{m}^{2}}{\lambda R_{B}}\right)
$$

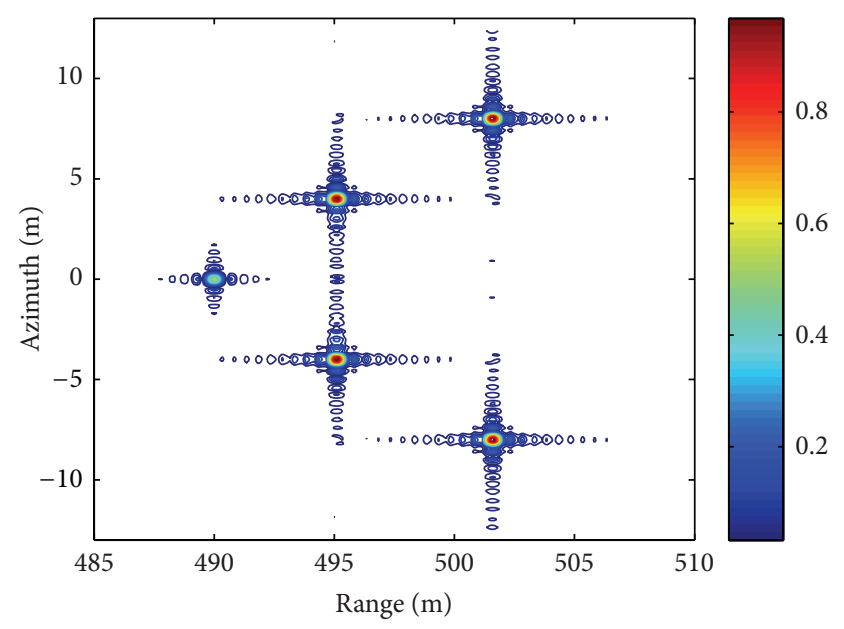

FIGURE 5: Azimuth and range 2D imaging results of the center antenna element.

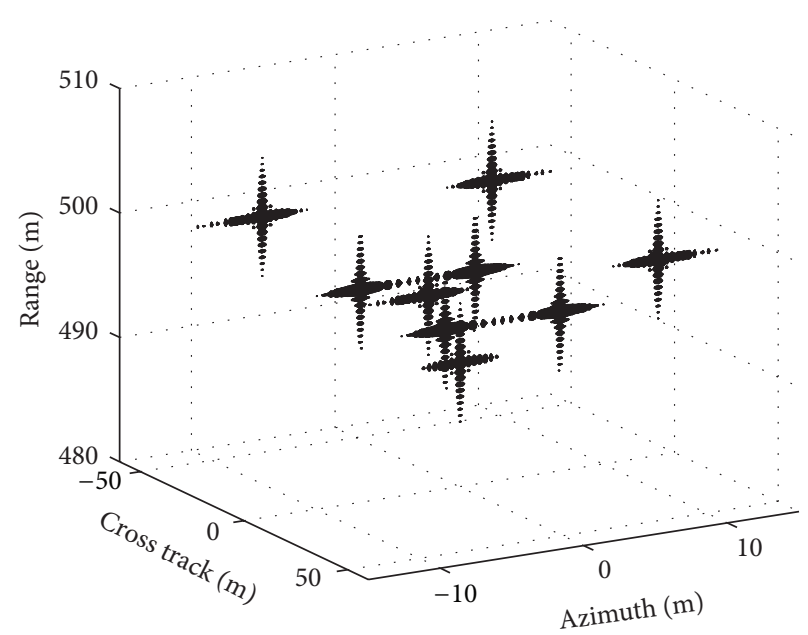

FIGURE 6: Final 3D image of down-looking sparse array SAR.

Then a cross track direction Fourier transform is performed on each range gate to realize the cross track compression, and the focused range-cross track image can be expressed by

$$
\begin{aligned}
& s\left(\widehat{t}, f_{y} ; \tau=\tau_{0}\right) \\
& =\operatorname{sinc}\left[B_{r}\left(\hat{t}-\frac{2\left(H-z_{p}\right)}{c}\right)\right] \\
& \quad \times \operatorname{sinc}\left[L_{f}\left(f_{y}-\frac{2 y_{p}}{\lambda r_{p} \cos \varphi_{p}}\right)\right] \\
& \quad \times \exp \left[-j \frac{2 \pi y_{p}^{2}}{\lambda r_{p} \cos \varphi_{p}}\right] \exp \left(-j \frac{4 \pi r_{p} \cos \varphi_{p}}{\lambda}\right)
\end{aligned}
$$

where $L_{f}$ is the effective aperture in the cross track direction. 
TABLE 3: The image quality parameters of the selected point target.

\begin{tabular}{lccc}
\hline Image quality parameters & Azimuth $(\mathrm{dB})$ & Range $(\mathrm{dB})$ & Cross track $(\mathrm{dB})$ \\
\hline PSLR & -13.20 & -13.31 & -13.41 \\
ISLR & -10.1069 & -11.2014 & -11.2879 \\
\hline
\end{tabular}

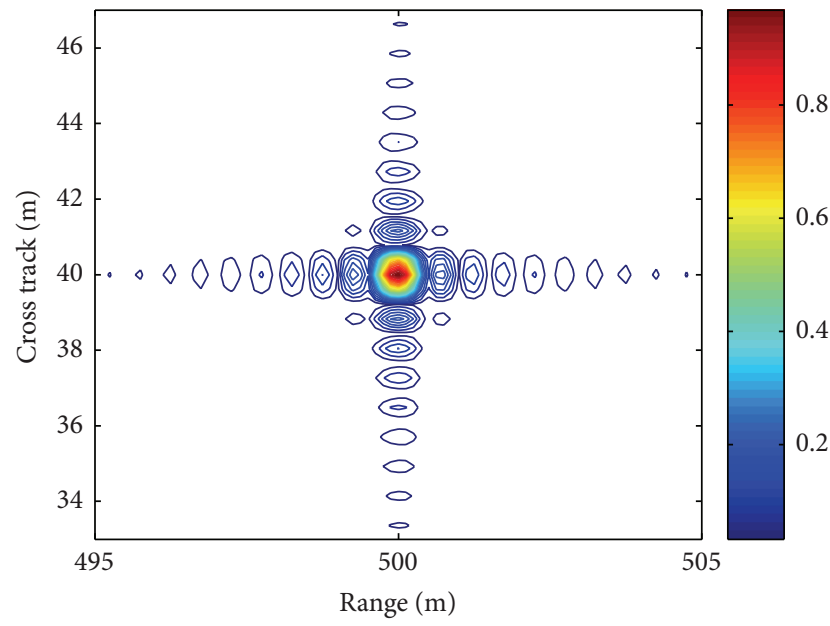

(a) Range-cross track section

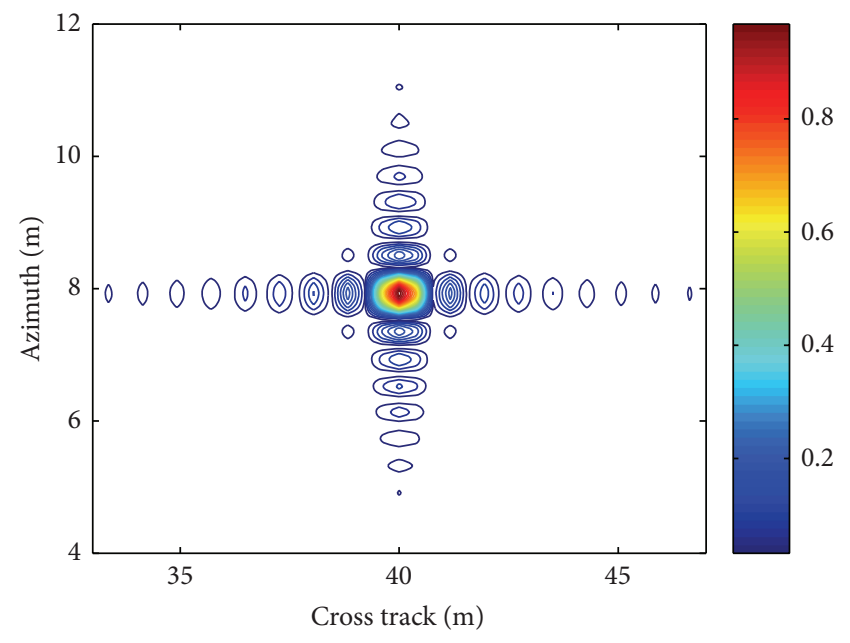

(b) Azimuth-cross track section

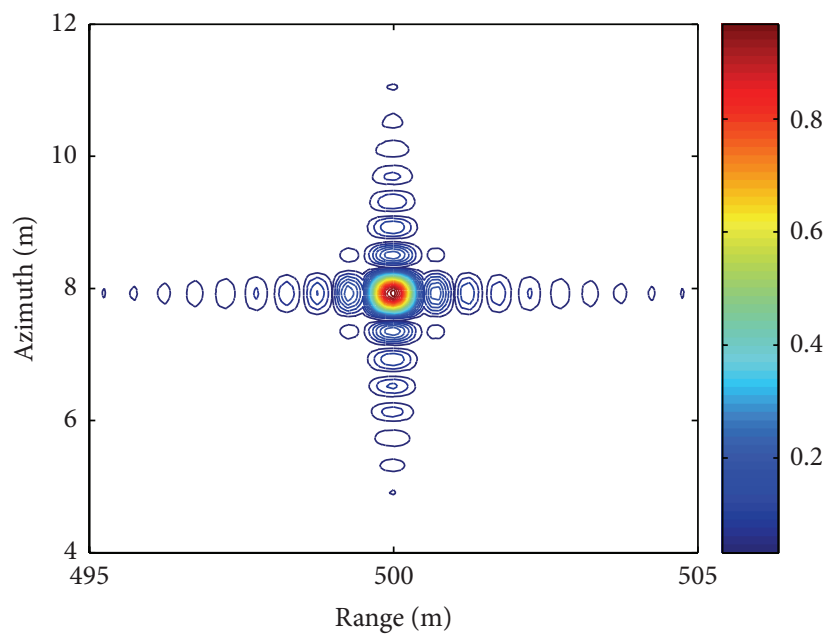

(c) Azimuth-range section

FIGURE 7:2D contour plots of the extracted point target.

From (22), it can be seen that the cross track resolution in the frequency domain is

$$
\Delta f_{y}=\frac{1}{L_{f}} .
$$

Moreover, based on Doppler characteristic analysis of the echo signal in SAR [19], the relationship between the cross track frequency and the cross track position of the point target can be written as

$$
f_{y p}=\frac{2}{\lambda} \sin \varphi_{p} \approx \frac{2 y_{p}}{\lambda\left(H-z_{p}\right)}
$$

Therefore, from (23) and (24), the cross track resolution can be calculated as

$$
\Delta y \approx \frac{\lambda\left(H-z_{p}\right)}{2 L_{f}} .
$$

From (25) we can get that the cross track resolution is not a constant, which varies with the vertical distance between the platform and the target.

When all the range-cross track sections corresponding to each azimuth position are processed following the procedure mentioned above, the 3D image of airborne down-looking array SAR can be achieved. From (8) and (22), we get that 


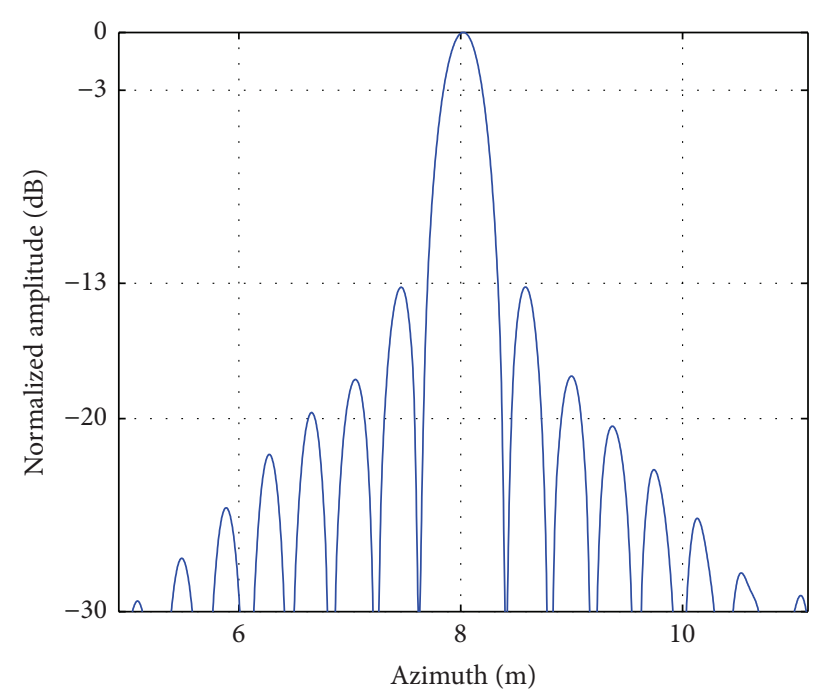

(a) The profile of the azimuth compression

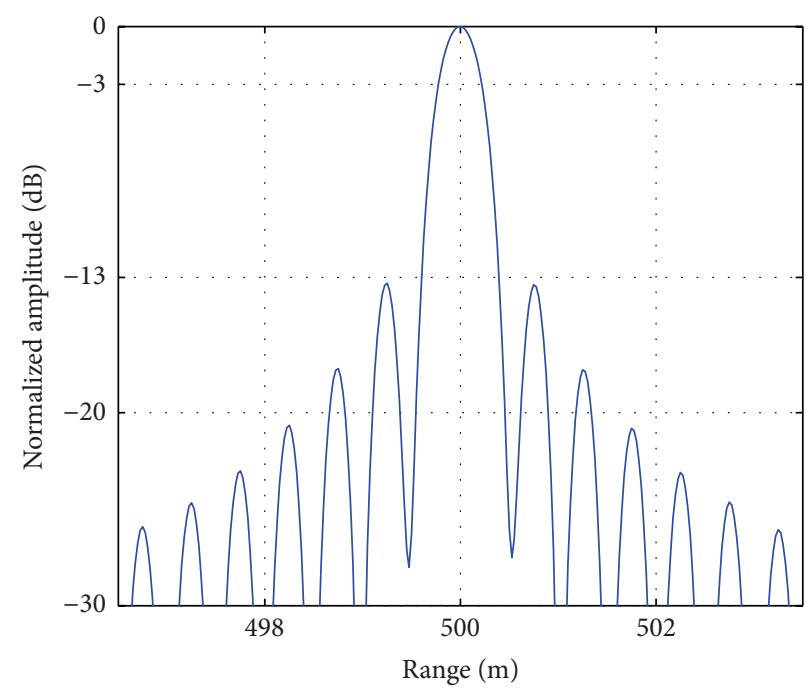

(b) The profile of the range compression

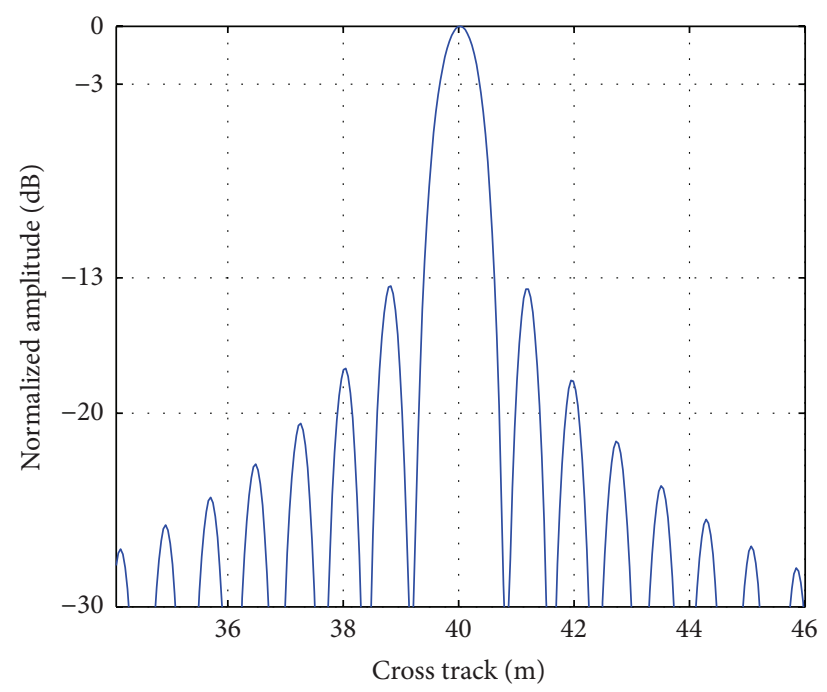

(c) The profile of the cross track compression

Figure 8: The profiles of the extracted point target.

the $3 \mathrm{D}$ point spread function of airborne down-looking array SAR can be denoted as

$$
\begin{aligned}
& \operatorname{psf}\left(\tau, \widehat{t}, f_{y}\right) \\
& \sim \operatorname{sinc}\left[B_{a}\left(\tau-\tau_{p}\right)\right] \operatorname{sinc}\left[B_{r}\left(\hat{t}-\frac{2\left(H-z_{p}\right)}{c}\right)\right] \\
& \quad \times \operatorname{sinc}\left[L_{f}\left(f_{y}-\frac{2 y_{p}}{\lambda r_{p} \cos \varphi_{p}}\right)\right] .
\end{aligned}
$$

\section{Simulation Results}

In this section, point target simulation is carried out to verify the validity of the proposed imaging algorithm. The main parameters used for simulation are listed in Table 1.
Here, we suppose that there are nine point targets located at the scene. The position parameters of the nine point targets are shown in Table 2. Figure 5 shows the azimuth and range $2 \mathrm{D}$ imaging results of the center antenna element. Because the point targets located symmetrically to the flight direction are imaged in one resolution cell, it can be seen that there are only five point targets that are shown in Figure 5 after the azimuth and range focusing.

With the 3D imaging processing by using the proposed algorithm in this paper, the surfaces of the final $3 \mathrm{D}$ image are plotted at $-30 \mathrm{~dB}$ in Figure 6. As expected, the image is reconstructed in 3D space, and the positions of the nine point targets are consistent with the real situation in Table 2. From Figure 6 we can get that the left/right ambiguity of the point targets caused by the symmetric range distances on both sides of the flight direction can be resolved after cross track imaging processing. 
In order to reflect the detailed characteristics of the imaging results, the point target located at $(8,40,500)$ is extracted for individual analysis. Figure 7 shows the contour plots of the imaging results of the extracted point target. Figure 7(a) shows the 2D image of the point target at the range-cross track section. Figure 7(b) shows the 2D image of the point target at azimuth-cross track section. Figure 7(c) shows the $2 \mathrm{D}$ image of the point target at azimuth-range section. From Figure 7, it is clear that the shape of the contour plots is regular, main lobes and side lobes visibly divided, indicating that the point target is focused well.

Then, the image quality parameters, that is, the peak sidelobe ratio (PSLR) and the integrated sidelobe ratio (ISLR), are used to test the performance and focusing quality of the proposed method. Taking the point target located at (8, $40,500)$ as an example, Figure 8 shows the profiles of the azimuth, range, and cross track compression. From the above simulation results, we can get that the compression results all present the shape of a sinc function. Table 3 gives the performance parameters of the point target. Both the PSLR and the ISLR are close to the ideal values.

\section{Conclusions}

Down-looking array SAR can reconstruct 3D images of the observed area and overcome restrictions of shading and lay over effects in side-looking SAR. Therefore, down-looking array SAR has challenging potential for 3D digital maps, complex terrain mapping, and so on. In this paper, a novel 3D imaging algorithm capable of focusing down-looking SAR data is proposed. The principle behind the method is based on a special squint model in cross track processing to obtain accurate focusing. And the phase compensation factors and algorithm realization procedure are demonstrated in detail. Moreover, the method requires only Fourier transform and multiplications, making it suitable for practical applications. Raw data of down-looking sparse array SAR is simulated and the $3 \mathrm{D}$ image is achieved. The results of the simulated data confirm the effectiveness of the proposed method.

\section{Conflict of Interests}

The authors declare that there is no conflict of interests regarding the publication of this paper.

\section{Acknowledgments}

This work was supported by the National Natural Science Foundation of China under Grant 61201390 and Grant 61201389, the Fundamental Research Funds for the Henan Provincial Colleges and Universities under Grant 2014YWQQ10, and the Plan for Young Backbone Teacher of Henan University of Technology.

\section{References}

[1] D. L. Mensa, High Resolution Radar Imaging, Artech House, Dedham, Mass, USA, 1981.
[2] W. G. Carrara, R. S. Goodman, and R. M. Majewski, Spotlight Synthetic Aperture Radar: Signal Processing and Algorithms, Artech House, Boston, Mass, USA, 1995.

[3] B. Wang, Y. P. Wang, W. Hong, W. Tan, and Y. Wu, "Studies on MB-SAR 3D imaging algorithm using Yule-Walker method," Science China: Information Sciences, vol. 53, no. 9, pp. 1848-1859, 2010.

[4] X. X. Zhu and R. Bamler, "Demonstration of super-resolution for tomographic SAR imaging in urban environment," IEEE Transactions on Geoscience and Remote Sensing, vol. 50, no. 8, pp. 3150-3157, 2012.

[5] C. H. Gierull, "On a concept for an airborne downwardlooking imaging radar," International Journal of Electronics and Communications, vol. 53, no. 6, pp. 295-304, 1999.

[6] J. Klare, "A new airborne radar for 3D imaging-simulation study of ARTINO," in Proceesings of the 6th European Conference on Synthetic Aperture Radar, Dresden, Germany, May 2006.

[7] L. Du, Y. P. Wang, W. Hong, W. Tan, and Y. Wu, "A threedimensional range migration algorithm for downward-looking 3D-SAR with single-transmitting and multiple-receiving linear array antennas," EURASIP Journal on Advances in Signal Processing, vol. 2010, Article ID 957916, 2010.

[8] X.-M. Peng, Y.-P. Wang, W.-X. Tan, W. Hong, and Y.-R. Wu, "Fast wavenumber domain imaging algorithm for airborne downward-looking array 3D-SAR based on region of interest pick," Journal of Electronics and Information Technology, vol. 35, no. 7, pp. 1525-1531, 2013.

[9] J. Klare, "Digital beamforming for a 3D mimo sar-improvements through frequency andwaveform diversity (IGARSS '08)," in Proceedings of the IEEE International Geoscience and Remote Sensing Symposium, pp. 17-20, Boston, Mass, USA, July 2008.

[10] Y. N. Hou, Study of radar imaging technology based on sparse array antenna [Ph.D. dissertation], Institute of Electronics, Chinese Academy of Sciences, 2010.

[11] J. F. Nouvel, H. Jeuland, G. Bonin et al., "A Ka band imaging radar: DRIVE on board ONERA motorglider," in Proceedings of the International Geoscience and Remote Sensing Symposium (IGARSS '06), pp. 134-136, Denver, Colo, USA, August 2006.

[12] L. Du, Y. P. Wang, and W. Hong, “Three-dimensional imaging algorithm for synthetic aperture radar with linear array antennas based on elevation angle compression principle," Journal of the Graduate School of the Chinese Academy of Sciences, vol. 27, no. 6, pp. 800-808, 2010.

[13] R. Giret, H. Jeuland, and P. Enert, "A study of a 3D-SAR concept for a millimeter wave imaging radar onboard an UAV," in Proceedings of the 1st European Radar Conference (EURAD '04), pp. 201-204, Amsterdam, The Netherlands, October 2004.

[14] X. Wen, G. Kuang, J. Hu, R. Zhan, and J. Zhang, "Forwardlooking imaging of scanning phased array radar based on the compressed sensing," Progress in Electromagnetics Research, vol. 143, pp. 575-604, 2013.

[15] X. Z. Ren, L. N. Chen, and J. Yang, "3D imaging algorithm for down-looking MIMO array SAR based on Bayesian compressive sensing," International Journal of Antennas and Propagation, vol. 2014, Article ID 612326, 9 pages, 2014.

[16] M. Weiß, J. Ender, O. Peters et al., "An airborne radar for three dimensional imaging and observation-technical realisation and status of ARTINO," in Proceedings of the 6th European Conference on Synthetic Aperture Radar, Dresden, Germany, May 2006. 
[17] M. Y. Jin and C. Wu, "A SAR correlation algorithm which accommodates large-range migration," IEEE Transactions on Geoscience and Remote Sensing, vol. 22, no. 6, pp. 592-597, 1983.

[18] M. Soumekh, Synthetic Aperture Radar Signal Processing with MATLAB Algorithms, Wiley-Interscience, New York, NY, USA, 1999.

[19] G. Lan and H. G. Frank, Digital Processing of Synthetic Aperture Radar Data Algorithms and Implementation, Artech House, 2005. 

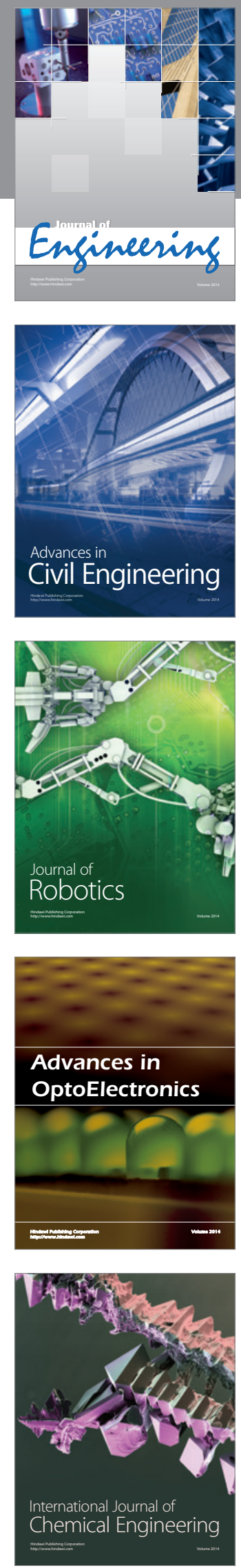

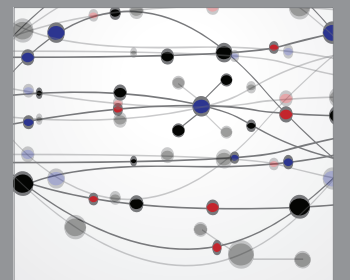

The Scientific World Journal
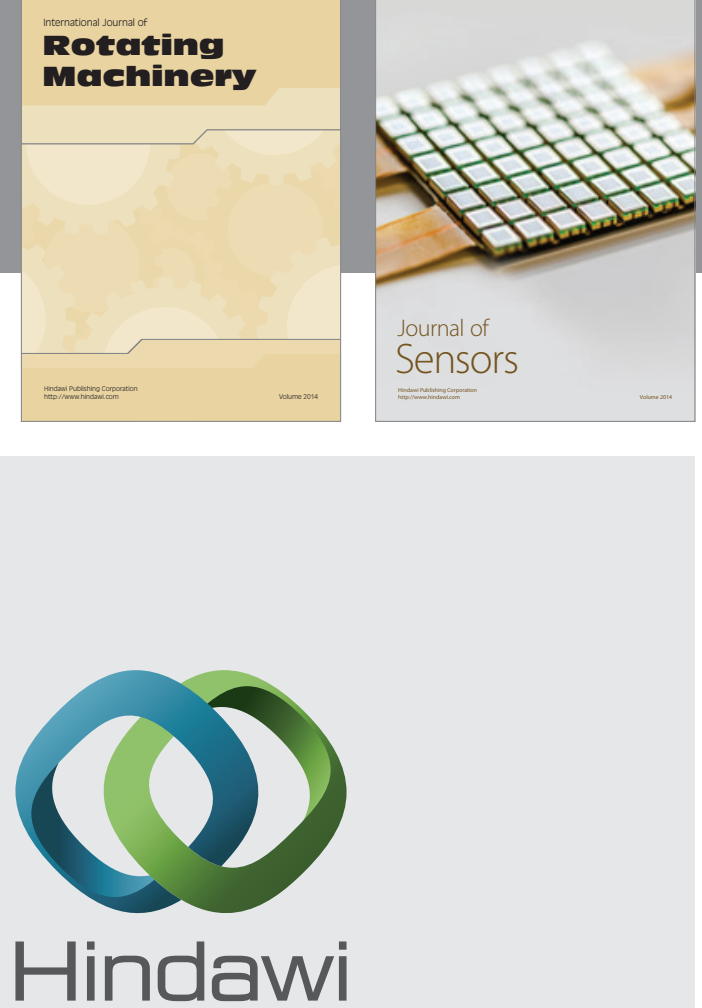

Submit your manuscripts at http://www.hindawi.com
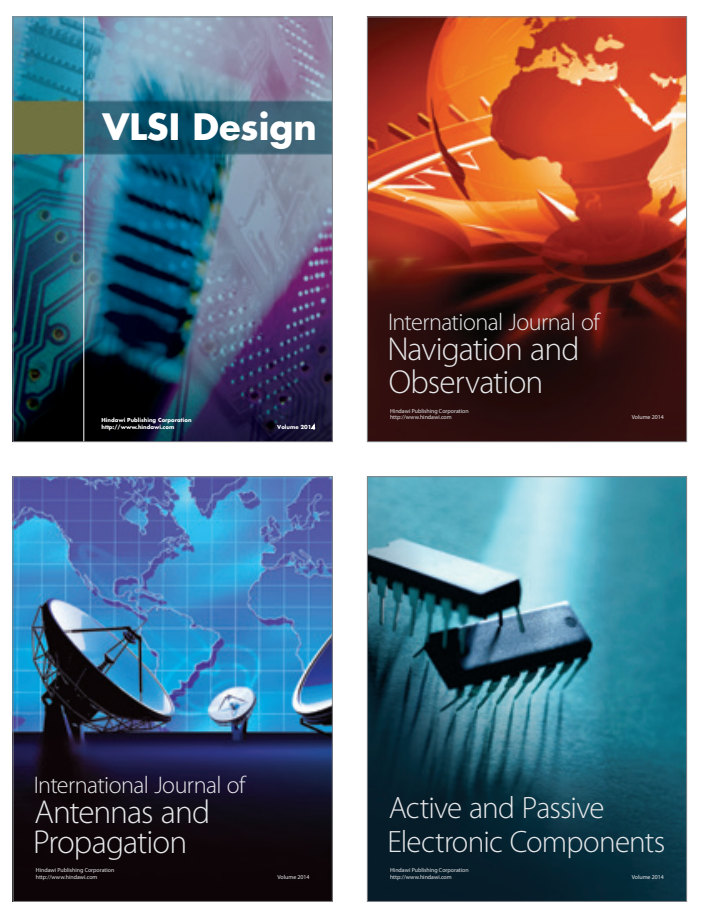
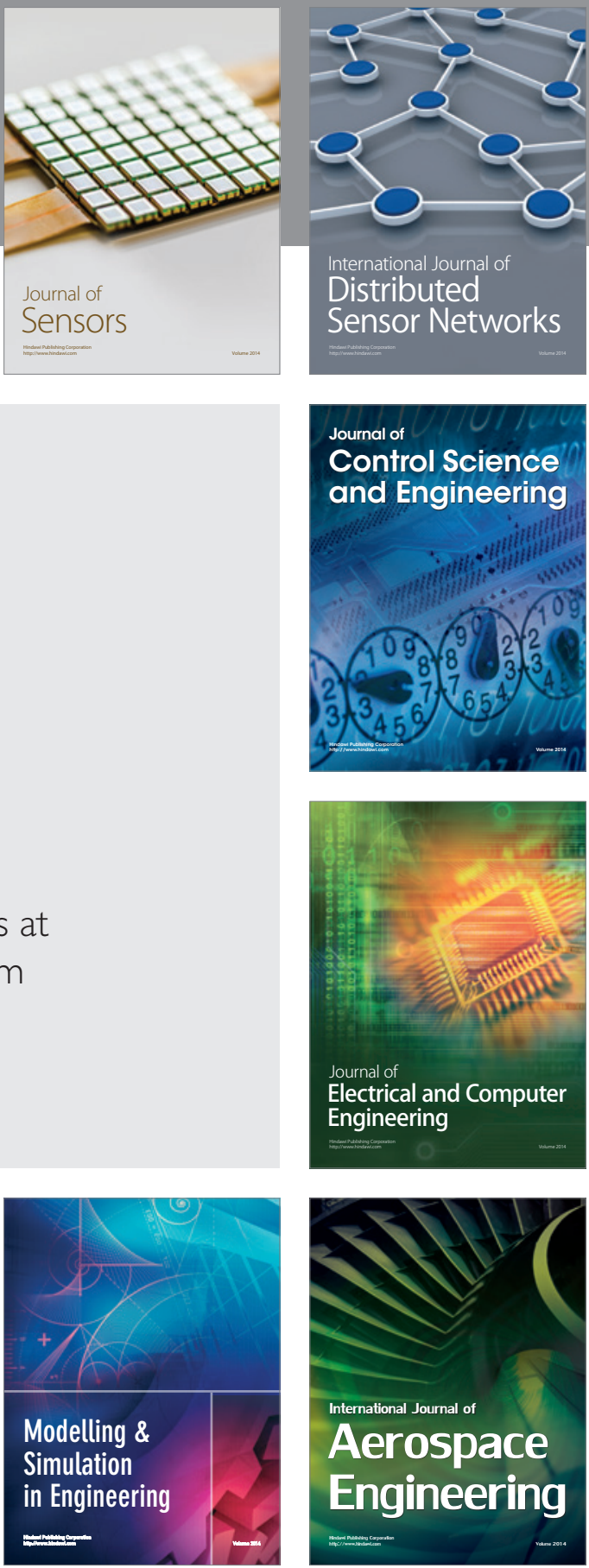

Journal of

Control Science

and Engineering
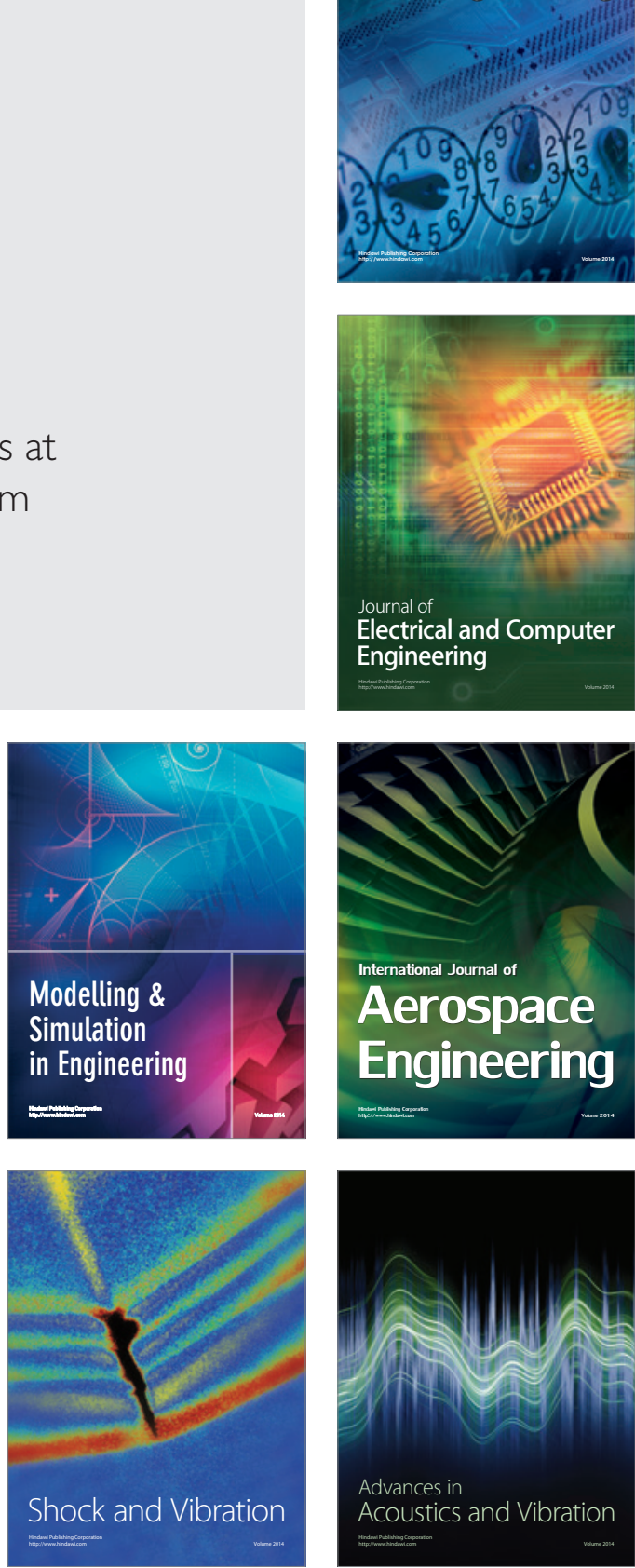